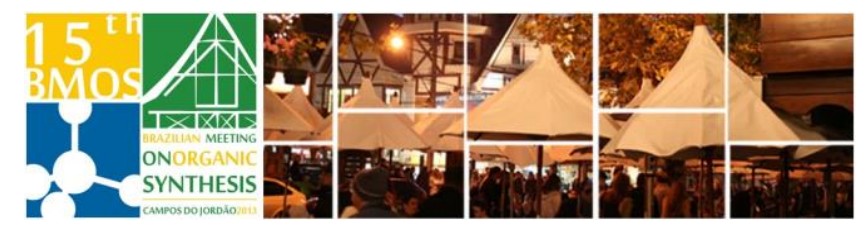

\title{
New phthalocyanine and azaphthalocyanine derivatives
}

\author{
Jan Svec, ${ }^{*}$ Petr Zimcik \\ Department of Pharmaceutical Chemistry and Drug Control, Faculty of Pharmacy in Hradec Kralove, Charles \\ University in Prague, Heyrovskeho 1203, Hradec Kralove, 500 05, Czech Republic \\ *svecjan2@faf.cuni.cz
}

Keywords: dyes, phthalocyanine, azaphthalocyanine,

\section{INTRODUCTION}

Phthalocyanines (Pcs) and azaphthalocyanines (AzaPcs) are analogues of porphyrin dyes where methine groups in bridge are replaced within nitrogens. ${ }^{1}$ Due to their promising spectral, photochemical, photophysical, and electrochemical properties, they are investigated for example as sensors, photosensitizers in photodynamic therapy or quenchers of fluorescence. Different cations coordinated in the center as well as different peripheral substitution of $\mathrm{Pc}$ and AzaPc influence significantly final spectral and photophysical properties.

\section{RESULTS AND DISCUSSION}

Cobalt is not typical central atom in Pcs and AzaPcs. Thus, we prepared new Pc $2 \boldsymbol{a}$ and AzaPc $\mathbf{2 b}$ containing $\mathrm{Co}_{\mathrm{o}}$ in the cavity and bearing $t$ butylsulfanyl groups on periphery (Figure 1). These macrocycles were prepared by cyclotetramerization of precursors 1a, 1b. Magnesium Pc and AzaPc were converted to the metal-free derivatives under acid conditions. Metal-free $\mathrm{Pc}$ and $\mathrm{AzaPc}$ reacted with anhydrous cobalt acetate to form cobalt complexes.
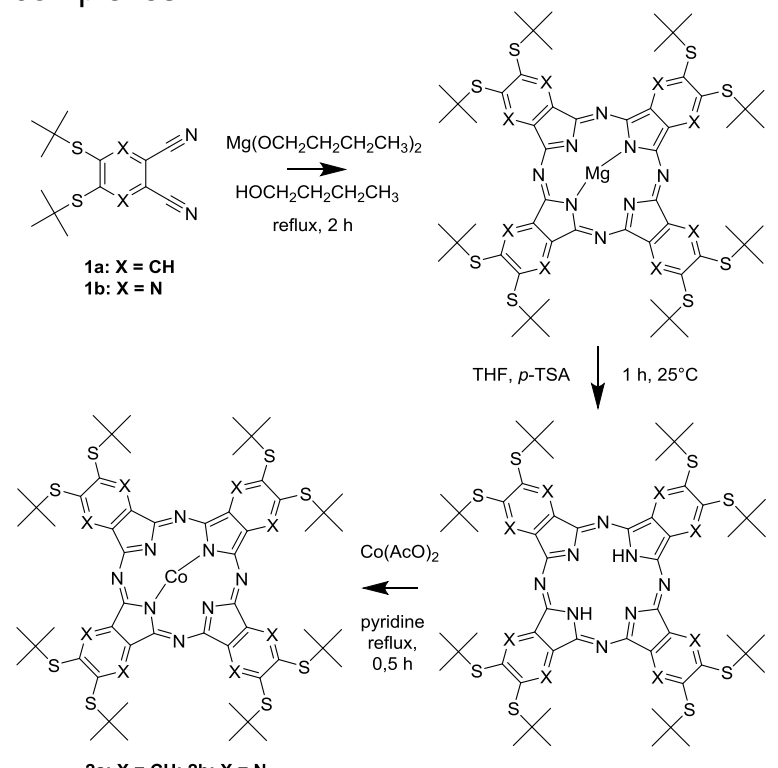

Figure 1. Synthesis of Co complexes of Pc and AzaPc
Preparation of water soluble zinc AzaPc 3 with quarternary ammonium groups on periphery (Figure 2) will be discussed in the second part of the work. Condition of synthesis and photodynamic activity on cancer cells will be also presented.

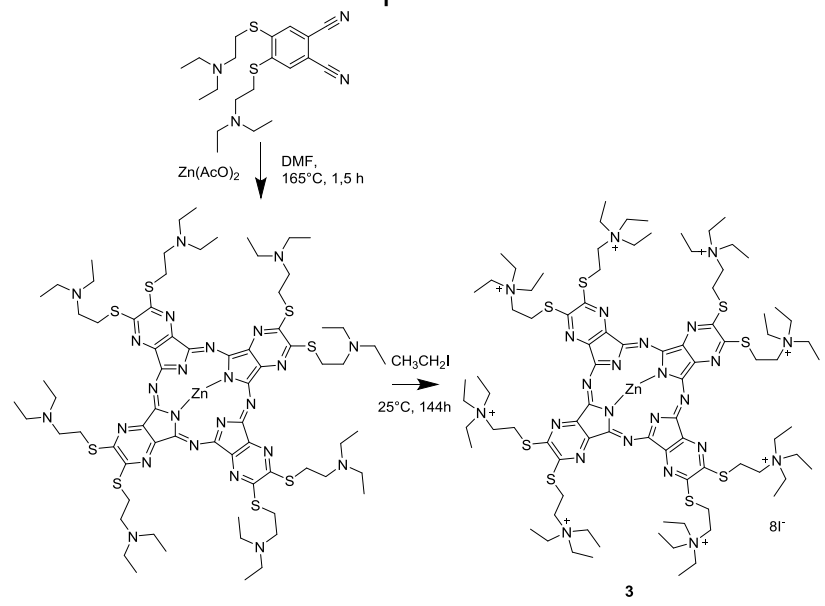

Figure 2. Synthesis of water soluble zinc AzaPc 3 containing quarternary ammonium groups on periphery.

\section{CONCLUSION}

New phthalocyanine and azaphthalocyanines coordinating $\mathrm{Co}$ in the central cavity and bearing $t$ butylsulfanyl groups on periphery were prepared and studied. Water soluble zinc AzaPc with quarternary ammonium groups on periphery were synthesized and photodynamic activity on cancer cells studied.

\section{ACKNOWLEDGEMENTS}

Project is co-financed by the European Social Fund and the state budget of the Czech Republic. Project no. CZ.1.07/2.3.00/30.0061.

\section{REFERENCES}

1 Kostka, M.; Zimcik, P.; Miletin M.; Klemera, P.; Kopecky K.; Musil, Z. Journal of Photochemistry and Photobiology A-Chemistry. 2006, 178, 16. 